\title{
Normas contínuas para a Escala de Estilos de Decisão (Decision Styles Scale) em uma amostra brasileira
}

Continuous norms for the Decision Styles Scale in a Brazilian Sample

Normas continuas para la escala de estilos de decisión en una muestra brasileña

André Luiz de Carvalho Braule Pinto https://orcid.org/0000-0001$\underline{9589-5756}$

Gabriel dos Santos Mouta - https://orcid.org/0000-0002-5796-9491

Sonia Regina Pasian - https://orcid.org/0000-0002-2811-6625

Leonardo Rodrigo Baldaçara - https://orcid.org/0000-0002-5201-8515

Antônio Geraldo da Silva - https://orcid.org/0000-0003-3423-7076

Leandro Fernandes Malloy-Diniz - https://orcid.org/0000-0002-6606$\underline{1354}$

\section{RESUMO:}

Introdução: A Escala de Estilos de Tomada de Decisão é um instrumento desenvolvido para avaliar dois estilos decisórios distintos, o intuitivo/heurístico vs. o deliberado/racional. Estudos anteriores verificaram que a adaptação brasileira da escala é adequada e apresenta boas propriedades psicométricas. O presente estudo apresenta parâmetros preliminares no formato de normas contínuas para 0 instrumento. Métodos: Participaram do estudo 1.218 adultos brasileiros com idade entre 18 e 64 anos. Resultados: Os resultados da escala não foram influenciados por idade ou sexo reportado, sendo possível apresentar os resultados em normas contínuas para a amostra global. Conclusão: Os resultados apresentados constituem-se em normas preliminares para a interpretação da versão brasileira do estudo. Novos estudos são necessários para a obtenção de normas com base em amostras representativas da população. 
Palavras chave: Tomada de decisão, processamento dual, adaptação transcultural, estilos de tomada de decisão.

\section{ABSTRACT:}

Introduction: The Decision Styles Scale (DSS) is an instrument developed to assess two distinct decision-making styles, the intuitive/heuristic vs. deliberate/rational. Previous studies found that the Brazilian Portuguese adaptation of the DSS was adequate and had good psychometric properties. This study presents preliminary parameters in the format of continuous norms for the scale. Methods: 1218 Brazilian adults aged from 18 to 64 years participated in the study. Results: The scale results were not influenced by reported age or sex, and the results can be presented as continuous norms for the entire sample. Conclusion: The results presented constitute preliminary norms for interpretation of the Brazilian version of the DSS. Future studies are needed to derive Brazilian norms based on representative samples of the population.

Keywords: Decision-making, dual processing theory, cross-cultural adaptation, decision-making styles.

\section{RESUMEN:}

Introducción: La Escala de Estilos de Decisión (EDE) es un instrumento desarrollado para evaluar dos estilos distintos de toma de decisiones, el intuitivo/heurístico frente al deliberado/racional. Estudios anteriores encontraron que la adaptación del DSS al portugués brasileño era adecuada y tenía buenas propiedades psicométricas. Este estudio presenta parámetros preliminares en el formato de normas continuas para la escala. Métodos: Participaron en el estudio 1218 adultos brasileños de entre 18 y 64 años. Resultados: Los resultados de la escala no fueron influenciados por la edad o el sexo reportado, y los resultados pueden ser presentados como normas continuas para toda la muestra. Conclusión: Los resultados presentados constituyen normas preliminares para la interpretación de la versión brasileña del DSS. Se necesitan estudios futuros para obtener normas brasileñas basadas en muestras representativas de la población. 
Palabras clave: Toma de decisiones, teoría del procesamiento dual, adaptación transcultural, estilos de toma de decisiones.

Como citar: Pinto, A.L.C.B; Mouta, G.; Pazian, S.; Baldaçara, L.R.; Silva, A.G.; Malloy-Diniz, L.F. - Normas contínuas para a Escala de Estilos de Decisão (Decision Styles Scale) em uma amostra brasileira. Debates em Psiquiatria, Rio de Janeiro, 2021; 11:1-16. https://doi.org/10.25118/2763-9037.2021.v11.211

\section{Conflito de interesses: declaram não haver}

Fonte de financiamento: declaram não haver

Parecer CEP: FFCLRP-USP-CAAE: 62744516.3.0000.5407- Número do Parecer: 1.991 .018

Recebido em: 04/05/2021

Aprovado em: 04/05/2021

Publicado em: 20/09/2021

\section{Introdução}

O estudo da tomada de decisão, por sua relevância na compreensão sobre como as pessoas selecionam alternativas, tem atraído a atenção de profissionais de diferentes áreas, como a filosofia, administração, economia, marketing, ciência dos esportes, políticas públicas, educação, psicologia e medicina [1]. A capacidade de analisar alternativas e selecionar uma ou mais opções em detrimento de outras é de fundamental importância para a adaptação e manutenção de um nível funcional adequado a diferentes contextos. Nesse sentido, o conceito de tomada de decisão refere-se ao processo de escolha entre alternativas concorrentes que apresentam consequências potencialmente diferentes. A concepção de que as decisões ocorrem de modo racional parte do pressuposto de que somos capazes de escolher deliberadamente as alternativas com maior potencial de ganho e menor custo. Essa concepção é a base dos modelos normativos da tomada de decisão [2] ], segundo o qual, na seleção entre alternativas, considerando todas as informações disponíveis, entram em jogo diferentes elementos, como o valor (importância que se dá a algum elemento de uma alternativa), a utilidade (o grau de satisfação/êxito potencialmente obtido a partir da alternativa escolhida) e a probabilidade 
(chance de obtenção de satisfação/êxito a partir da alternativa selecionada).

Ainda que a racionalidade tenha sido um elemento norteador dos estudos clássicos sobre a tomada de decisão, essa concepção tem sido amplamente questionada ao longo do tempo. Os processos decisórios são influenciados por uma série de variáveis subjetivas/irracionais que podem levar a escolhas que desconsideram a análise deliberada do custo e do benefício em termos temporais (consequências imediatas vs. consequências de longo prazo). Variáveis situacionais, como distância da decisão em relação

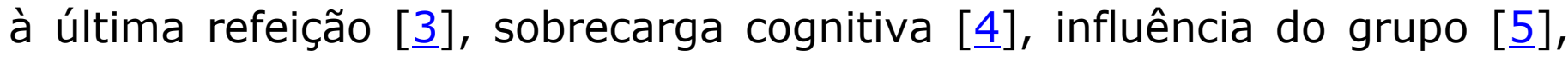
escassez/abundância de recursos $[\underline{6}, \underline{7}]$, podem modular escolhas sem que tenhamos a percepção exata dessa influência. Além disso, vieses cognitivos podem influenciar de forma substancial um julgamento e uma decisão, fugindo à expectativa de racionalidade (vide, por exemplo, o efeito de framing descrito por Kaneman \& Tversky) []].

Além da influência das variáveis situacionais, aspectos relativamente estáveis da personalidade e cognição de um indivíduo podem subsidiar tendências e estilos decisórios. Estudos com pacientes com comprometimentos em sistemas neurais específicos mostram que esses indivíduos tendem a decidir de modo previsível. Por exemplo, comprometimentos em circuitos que envolvem o córtex orbitofrontal podem levar a estilos decisórios mais imediatistas com prejuízos na antecipação de cenários [ㅁ] . Por outro lado, em transtornos psiquiátricos, padrões imediatistas/não prospectivos de tomada de decisão são encontrados em indivíduos com transtorno do déficit de atenção e hiperatividade [10], transtorno obsessivo-compulsivo [1ㅣ], transtorno bipolar [12], alcoolismo [13], transtorno de personalidade borderline [14], entre outras condições clínicas. A tomada de decisão imediatista pode estar relacionada a diversos desfechos clínicos adversos, como as tentativas de suicídio $[\underline{12}, \underline{15}]$ e problemas financeiros [투 $]$.

Em contextos não clínicos, as dificuldades em tomar decisão considerando ganhos de longo prazo e com má gestão de risco também parecem estar relacionadas a problemas em múltiplas áreas, como comportamento no trânsito [17], problemas financeiros [18], prática de bullying no contexto escolar [19] e evasão escolar [20]. A hipótese de que as pessoas desenvolvem tendências em tomar decisões de maneira semelhante em diferentes situações da vida ao longo do tempo tem subsidiado a ideia de que há diferentes estilos decisórios, os quais influenciariam a forma como

4 Debates em Psiquiatria, Rio de Janeiro, 2021; 11:1-16

https://doi.org/10.25118/2763-9037.2021.v11.211

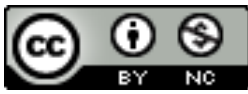


as pessoas resolvem problemas, julgam situações e fazem suas escolhas. Hamilton et al. [21] sugerem que esses estilos seriam a confluência entre traços de personalidade e a experiência/aprendizagem.

Dada a importância da tomada de decisão e o seu impacto em diferentes aspectos das atividades humanas, o desenvolvimento de medidas de avaliação desses processos tem recebido particular atenção nas últimas décadas. Medidas neuropsicológicas de tomada de decisão têm sido amplamente empregadas nesse sentido. No entanto, no Brasil, há poucos instrumentos adaptados e com resultados favoráveis que permitam uso para fins de pesquisa e outras aplicações (por exemplo, o Melbourne Decision-Making Questionnaire) [22]. No entanto, não há, até o momento, parâmetros normativos para o uso de escalas que avaliam os diferentes estilos de tomada de decisão para indivíduos brasileiros.

Recentemente, Mouta et al. [23] realizaram a adaptação transcultural da Escala de Estilos de Decisão (Decision Styles Scale - DSS) para a população brasileira. A escala é composta por 10 itens que apresentam situações em que as pessoas devem, a partir de uma escala Likert de 5 pontos, reportar sobre suas tendências decisórias. Os resultados da escala são úteis para informar sobre dois estilos de tomada de decisão: o racional (ex.: análise deliberada de prós e contras de uma determinada situação) e o intuitivo (ex.: decisões com base em impressões e sentimentos). Desde o seu desenvolvimento [21], diversos estudos têm reportado suas propriedades psicométricas, além de sua aplicabilidade na pesquisa sobre fenômenos sociais como a persuasão [24], autocontrole e comportamento

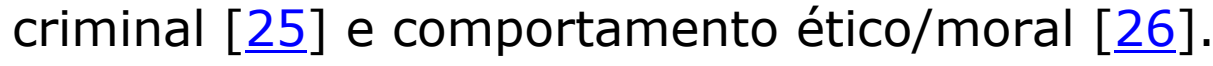

No estudo da adaptação transcultural da DSS para a população brasileira, Mouta et al. [23] reportaram dados que sustentam sua estrutura de dois fatores. Os resultados também apontaram propriedades psicométricas adequadas para o uso da escala em nosso contexto. No entanto, o estudo não apresenta parâmetros para a interpretação da escala para uso no Brasil. O objetivo do presente estudo é apresentar parâmetros para uso da escala quando aplicada em indivíduos brasileiros.

\section{Método}

Participantes

Os participantes foram 1.218 indivíduos selecionados por conveniência, $73,4 \%$ do sexo feminino $(n=816)$, com idade entre 18 e 64 anos $(M=$ $26,68 ; \mathrm{DP}=8,32$ ) e escolaridade média de 16,86 anos ( $\mathrm{DP}=3,72)$. Em 
relação ao estado civil, 818 referiram ser solteiros $(67,15 \%)$, de classe social alta (51,64\% entre as classes B2 e A). A Tabela 1 apresenta as características sociodemográficas detalhadas.

\begin{tabular}{|c|c|c|c|c|c|c|c|}
\hline Variáveis & f & $\%$ & $\mathbf{n}$ & Média & DP & Min & Max \\
\hline \multicolumn{8}{|l|}{ Regiões do Brasil } \\
\hline Centro-Oeste & 17 & 1,5 & & & & & \\
\hline Nordeste & 54 & 4,9 & & & & & \\
\hline Norte & 856 & 77,0 & & & & & \\
\hline Sudeste & 156 & 14,0 & & & & & \\
\hline Sul & 28 & 2,5 & & & & & \\
\hline \multicolumn{8}{|l|}{ Sexo } \\
\hline Feminino & 816 & 73,4 & & & & & \\
\hline Masculino & 295 & 26,6 & & & & & \\
\hline Idade & & & 936 & 26,7 & 8,32 & 18 & 64 \\
\hline Escolaridade & & & 962 & 16,9 & 3,72 & 8,00 & 29,0 \\
\hline $\begin{array}{l}\text { Classe } \\
\text { socioeconômica } \\
\text { Escala de estilo de } \\
\text { decisão }\end{array}$ & & & 1110 & 31,9 & 10,96 & 7 & 79 \\
\hline Racional & & & 1218 & 20,7 & 3,63 & 5 & 25 \\
\hline Intuitivo & & & 1218 & 14,8 & 3,98 & 5 & 25 \\
\hline
\end{tabular}

\section{Instrumento: Decision Styles Scale (DSS)}

Desenvolvida por Hamilton et al. [21] e adaptada para o português brasileiro por Mouta et al. [23] , é uma medida de autorrelato que avalia o estilo racional ("Eu considero muitos fatores quando tomo decisões") e o estilo intuitivo ("Quando tomo decisões, eu confio principalmente nos meus instintos"). A escala contém 10 itens e apresenta uma série de afirmações sobre as formas pelas quais as pessoas preferem tomar decisões, marcando em uma escala Likert de 5 pontos, entre 1 (discordo totalmente) a 5 (concordo totalmente). A versão usada neste estudo mostra excelente adequação [comparative fit indices $(\mathrm{CFI})=0,937$; root mean squared error of approximation (RMSEA) $=0,076 ; \mathrm{p}<0,001 ; 90 \%$ confidence interval $(90 \% \mathrm{CI})=0,066-0,086 ;$ Tucker-Lewis index $(\mathrm{TLI})=0,917$; standardized root mean square residual $(S R M R)=0,073$ ] e alta confiabilidade acessada pelo alfa de Cronbach e coeficiente de McDonald para estilo racional ( $a=$ 0,832 e $\omega=0,837$ ) e para estilo intuitivo ( $a=0,770$ e $\omega=0,779$ ). 


\section{Procedimentos}

O estudo originalmente foi aprovado pelo Comitê de Ética em Pesquisa. Uma plataforma online foi desenvolvida e divulgada nas redes sociais. Após a leitura dos objetivos do estudo e a confirmação do consentimento por meio do Termo de Consentimento Livre e Esclarecido, os participantes preencheram a DSS em conjunto com instrumentos que avaliavam processos afetivos e um questionário sociodemográfico, com duração média de 15 minutos. Os dados apresentados no presente estudo referemse aos dados da DSS. As análises estatísticas foram realizadas através do software Jamovi27.

Primeiramente, foram realizadas análises de regressão linear, usando estimador Mínimos Quadrados Ordinários [ordinary least squares (OLS)], um tipo de método para estimar os parâmetros desconhecidos em um modelo de regressão linear. Em seguida, foram calculadas as normas para cada fator usando os procedimentos de normas contínuas sugeridos por Lenhard et al. $[\underline{28}, \underline{29}]$ e Lenhard \& Lenhard. [이

\section{Resultados}

Em relação aos participantes de diferentes regiões do país, observou-se participação das cinco regiões, com participação maior das regiões Norte e Sudeste, respectivamente. Em sua maioria, a amostra foi composta por mulheres, com alta escolaridade e poder aquisitivo. Nos escores médios da DSS, observa-se que os participantes reportaram maiores níveis para o estilo racional quando comparado ao estilo intuitivo.

Ajustamos um modelo linear (usando estimador OLS) para prever cada fator de DSS a partir do sexo e idade dos participantes. O modelo global não apresentou significância estatística nem para o estilo racional [R2 = $0,0012 ; \mathrm{F}(2,933)=0,59 ; \mathrm{p}=0,552] ;$ nem para o estilo intuitivo $[R 2=$ $0,007 ; F(2,933)=2,51 ; p=0,082]$. Dado que os modelos não apresentaram influência, nem do sexo, nem da idade, calculamos as normas com base exclusivamente na amostra total para cada fator (Tabela 2). As pontuações $T$ padronizadas desta tabela podem ser usadas para comparar a pontuação DSS de um indivíduo com as pontuações do grupo de referência. 
Tabela 2 - Dados normativos para cada fator da DSS estimados

a partir de uma amostra brasileira $(n=1.218)$

\section{Estilo racional Estilo intuitivo}

\begin{tabular}{lccccc}
\hline Escore & Norma & Percentil & Escore & Norma & Percentil \\
\hline 5,00 & 20,0 & 0,135 & 5,00 & 24,3 & 0,510 \\
6,00 & 21,3 & 0,208 & 6,00 & 27,7 & 1,276 \\
7,00 & 22,9 & 0,332 & 7,00 & 30,6 & 2,615 \\
8,00 & 24,4 & 0,520 & 8,00 & 33,3 & 4,740 \\
9,00 & 25,9 & 0,801 & 9,00 & 35,9 & 7,861 \\
10,00 & 27,5 & 1,213 & 10,00 & 38,3 & 12,165 \\
11,00 & 29,1 & 1,812 & 11,00 & 40,8 & 17,770 \\
12,00 & 30,7 & 2,669 & 12,00 & 43,2 & 24,697 \\
13,00 & 32,4 & 3,880 & 13,00 & 45,6 & 32,829 \\
14,00 & 34,1 & 5,566 & 14,00 & 48,0 & 41,902 \\
15,00 & 35,9 & 7,880 & 15,00 & 50,4 & 51,508 \\
16,00 & 37,7 & 11,005 & 16,00 & 52,8 & 61,137 \\
17,00 & 39,7 & 15,151 & 17,00 & 55,3 & 70,243 \\
18,00 & 41,8 & 20,541 & 18,00 & 57,8 & 78,336 \\
19,00 & 44,0 & 27,382 & 19,00 & 60,4 & 85,064 \\
20,00 & 46,4 & 35,813 & 20,00 & 63,0 & 90,277 \\
21,00 & 49,0 & 45,834 & 21,00 & 65,6 & 94,028 \\
22,00 & 51,8 & 57,205 & 22,00 & 68,2 & 96,533 \\
23,00 & 55,1 & 69,344 & 23,00 & 70,7 & 98,088 \\
24,00 & 58,9 & 81,270 & 24,00 & 73,2 & 98,990 \\
25,00 & 63,8 & 91,695 & 25,00 & 75,7 & 99,484 \\
\hline
\end{tabular}

\section{Discussão}

Os resultados apresentados neste estudo caracterizam o desempenho de uma amostra brasileira na escala DSS e constituem-se como o primeiro estudo com parâmetros de interpretação obtidos a partir de dados coletados com amostras de população geral. Esses resultados complementam os achados de Mouta et al. [23], que reportaram a adaptação transcultural e propriedades psicométricas da versão brasileira da escala. No estudo, os autores encontraram indicadores psicométricos semelhantes ao estudo original da escala, e a adequação da versão adaptada serviu de base para a análise dos parâmetros preliminares de interpretação aqui reportados. 
Desde o desenvolvimento inicial da escala, a DSS foi submetida a estudos que aferiram suas propriedades psicométricas. Hamilton et al. [21] verificaram a estrutura fatorial que suporta a presença dos dois estilos fatoriais, racional e intuitivo. Nesse mesmo estudo, os autores apresentaram excelentes indicadores de confiabilidade e fidedignidade da escala, além de dados que suportam a validade divergente e discriminante do instrumento quando comparado com outras medidas psicológicas de personalidade. Posteriormente, os mesmos autores verificaram que os estilos de personalidade aferidos pela escala são preditivos de fenômenos distintos [31].

Enquanto o estilo racional de decisão foi positivamente correlacionado com a precisão e velocidade das decisões, a criatividade e a confiança se relacionam com informações baseadas em estados afetivos no processo decisório. Por outro lado, o estilo intuitivo relacionou-se positivamente com a confiança nos estados afetivos em processos decisórios e negativamente com o aprofundamento em informações para nortear decisões. Os autores também demonstraram potenciais interações entre esses dois estilos na velocidade e eficiência da tomada de decisão.

De acordo com a proposta original, a DSS é compatível com os modelos teóricos de processamento dual que postulam a existência da dicotomia entre processos deliberados/controlados vs. processos automáticos/heurísticos. Nesse sentido, a DSS tem sido usada para avaliar processos intuitivos e deliberados relacionados à persuasão [32], maximização de ganhos em processos decisórios [33], comportamento/decisões éticas [31] e comportamento criminal [225]. Sua potencial aplicabilidade para a investigação de questões clínicas também é destacada pelos autores da escala.

Até onde sabemos, a adaptação brasileira da DSS foi a primeira e única até o momento desde a publicação da versão original [23]. A ausência de outros parâmetros normativos para a escala dificulta a comparação entre os nossos dados e os das outras versões do instrumento em contextos culturais distintos. Estudos futuros poderão propiciar tais comparações. Além das propriedades psicométricas reportadas por Mouta et al. [23] e dos parâmetros normativos preliminares aqui reportados, são necessários novos estudos para aferir a validade de critério e de construto da versão brasileira da DSS. 
O estudo apresenta uma importante limitação. Em que pese a amostra tenha sido composta por 1.218 participantes, não foram controladas variáveis sociodemográficas como região, sexo e escolaridade dos participantes. Estudos futuros deverão obter normas estratificadas para a escala considerando tais variáveis.

Apesar das limitações do presente estudo, que devem ser consideradas em análises utilizando o instrumento, os dados aqui reportados poderão ser utilizados para interpretação inicial dos resultados da versão brasileira do instrumento, principalmente em indivíduos com características semelhantes aos que compuseram a amostra dessa investigação. 


\section{Referências}

1. Schiavon B, Sallum I, Grassi-Oliveira R, Malloy-Diniz L.

Julgamento e tomada de decisão: conceitos gerais. In: Malloy-Diniz L, Klume-Schiavon B, Grassi-Oliveira R. Julgamento e tomada de decisão. São Paulo: Pearson; 2018. p. 25-46.

2. Arvai J, Campbell-Arvai V, Steel P. Decision-making for sustainability: a systematic review of the body of knowledge [Internet]. 2012 [cited 2021 Jul 19].

https://static1.squarespace.com/static/5d5156083138fd000193c11a /t/5f2efe6e0c19916a4256753a/1596915324664/NBS-SystematicReview-Decision-Making.pdf

3. Danziger S, Levav J, Avnaim-Pesso L. Extraneous factors in judicial decisions. Proc Natl Acad Sci U S A. 2011;108:6889-92. https://doi.org/10.1073/pnas.1018033108 https://doi.org/10.1073/pnas.1112190108 - PMid:21482790

4. Osgood JM. Ego-depletion increases selfish decision making, but may also increase self-conflict and regret about those decisions. J Soc Psychol. 2019;159:417-30.

https://doi.org/10.1080/00224545.2018.1505706 - PMid:30142302

5. Gong X, Sanfey AG. Social rank and social cooperation: impact of social comparison processes on cooperative decision-making. PLoS One. 2017; 12 :e0175472.

https://doi.org/10.1371/journal.pone.0175472 - PMid:28388684 PMCid:PMC5384784

6. Mani A, Mullainathan S, Shafir E, Zhao J. Poverty impedes cognitive function. Science. 2013;341:976-80.

https://doi.org/10.1126/science.1238041 - PMid:23990553

7. Ong Q, Theseira W, Ng IY. Reducing debt improves psychological functioning and changes decision-making in the poor. Proc Natl Acad Sci U S A. 2019;116:7244-9.

https://doi.org/10.1073/pnas.1810901116 - PMid:30910964 PMCid:PMC6462060 
8. Kahneman D, Slovic $P$, Tversky A. Judgment under uncertainty: heuristics and biases. Cambridge: Cambridge University; 1982. https://doi.org/10.1017/CB09780511809477

9. Howard JD, Kahnt T. Causal investigations into orbitofrontal control of human decision making. Curr Opin Behav Sci. 2021;38:14-9. https://doi.org/10.1016/j.cobeha.2020.06.013 PMid:32864400

10. Malloy-Diniz L, Fuentes D, Leite WB, Correa H, Bechara A. Impulsive behavior in adults with attention deficit/hyperactivity disorder: characterization of attentional, motor and cognitive impulsiveness. J Int Neuropsychol Soc. 2007;13:693-8. https://doi.org/10.1017/S1355617707070889 - PMid:17521490

11. Grassi G, Pallanti S, Righi L, Figee M, Mantione M, Denys D, Piccagliani D, Rossi A, Stratta P. Think twice: impulsivity and decision making in obsessive-compulsive disorder. J Behav Addict. 2015;4:263-72. https://doi.org/10.1556/2006.4.2015.039 PMid:26690621 PMCid:PMC4712760

12. Malloy-Diniz LF, Neves FS, Abrantes SS, Fuentes D, Corrêa H. Suicide behavior and neuropsychological assessment of type I bipolar patients. J Affect Disord. 2009;112:231-6. https://doi.org/10.1016/j.jad.2008.03.019 - PMid: 18485487

13. Camchong J, Endres M, Fein G. Decision making, risky behavior, and alcoholism. Handb Clin Neurol. 2014;125:227-36.

https://doi.org/10.1016/B978-0-444-62619-6.00014-8 PMid:25307578

14. Svaldi J, Philipsen A, Matthies S. Risky decision-making in borderline personality disorder. Psychiatry Res. 2012;197:112-8. https://doi.org/10.1016/j.psychres.2012.01.014 - PMid:22421066

15. Perrain R, Dardennes $R$, Jollant F. Risky decision-making in suicide attempters, and the choice of a violent suicidal means: an updated meta-analysis. J Affect Disord. 2021;280:241-9. https://doi.org/10.1016/j.jad.2020.11.052 - PMid:33220560 
16. Bangma DF, Koerts J, Fuermaier AB, Mette C, Zimmermann $M$, Toussaint AK, et al. Financial decision-making in adults with ADHD. Neuropsychology. 2019;33:1065-77. https://doi.org/10.1037/neu0000571 - PMid:31343233

17. Cheng AS, Ting $\mathrm{KH}$, Liu KP, Ba Y. Impulsivity and risky decision making among taxi drivers in Hong Kong: an event-related potential study. Accid Anal Prev. 2016;95:387-94. https://doi.org/10.1016/j.aap.2015.12.021 - PMid:26748873

18. Teichman JM, Matsumoto $E$, Smart M, Smith AE, Tongco W, Hosking $D E$, et al. Personal finances of residents at three Canadian universities. Can J Surg. 2005;48:27-32.

19. Medeiros W, Torro-Alves N, Malloy-Diniz LF, Minervino CM. Executive functions in children who experience bullying situations. Front Psychol. 2016;7:1197. https://doi.org/10.3389/fpsyg.2016.01197 - PMid:27616998 PMCid:PMC5000580

20. Bradshaw CP, O'Brennan LM, McNeely CA. Core competencies and the prevention of school failure and early school leaving. New Dir Child Adolesc Dev. 2008;122:19-32.

https://doi.org/10.1002/cd.226 - PMid:19021248

21. Hamilton K, Shih SI, Mohammed S. The development and validation of the rational an intuitive decision styles scale. J Pers Assess. 2016;98:523-35.

https://doi.org/10.1080/00223891.2015.1132426 - PMid:26967981

22. Cotrena C, Branco LD, Fonseca RP. Adaptation and validation of the Melbourne decision making questionnaire to Brazilian Portuguese. Trends Psychiatry Psychother. 2018;40:29-37. https://doi.org/10.1590/2237-6089-2017-0062 - PMid:29211117

23. Mouta GS, Pinto AL, Malloy-Diniz LF, Pasian RS. Cross-cultural adaptation, and factor structure of the decision styles scale for Brazil. Curr Res Behav Sci. 2021;2:100039. https://doi.org/10.1016/j.crbeha.2021.100039 
24. Wang X, Shi W, Kim R, Oh Y, Yang S, Zhang J, et al. Persuasion for good: towards a personalized persuasive dialogue system for social good. In: Proceedings of the 57th Annual Meeting of the Association for Computational Linguistics [Internet]. Stroudsburg: Association for Computational Linguistics. 2019 [cited 2021 Jul 29]. p. 5635-49. https://www.savethechildren.org/ https://aclanthology.org/P19-1566/ - PMCid:PMC6507442

25. McClanahan W, van der Linden S, Ruggeri K. Decision-making style mediates the relationship between trait self-control and selfreported criminal behavior. Pers Individ Dif. 2019;151:109537. https://doi.org/10.1016/j.paid.2019.109537

26. Tzini $\mathrm{K}$, Jain $\mathrm{K}$. Unethical behavior under relative performance evaluation: evidence and remedy. Hum Resour Manage.

2018;57:1399-413. - https://doi.org/10.1002/hrm.21913

27. The jamovi project. Jamovi [Internet]. 2021. https://www.jamovi.org

28. Lenhard A, Lenhard W, Suggate S, Segerer R. A continuous solution to the norming problem. Assessment. 2018;25:112-25. https://doi.org/10.1177/1073191116656437 - PMid:27371826

29. Lenhard A, Lenhard W, Gary S. Continuous norming of psychometric tests: a simulation study of parametric and semiparametric approaches. PLoS One. 2019;14: e0222279. https://doi.org/10.1371/journal.pone.0222279 - PMid:31527877 PMCid:PMC6748442

30. Lenhard W, Lenhard A. Improvement of norm score quality via regression-based continuous norming. educational and psychological measurement. Educ Psychol Meas. 2021;81:229-61. https://doi.org/10.1177/0013164420928457

31. Hamilton K, Shih SI, Mohammed S. The predictive validity of the decision styles scale: an evaluation across task types. Pers Individ Dif. 2017;119:333-40. https://doi.org/10.1016/j.paid.2017.08.009 
32. Parsons K, Butavicius M, Delfabbro P, Lillie M. Predicting susceptibility to social influence in phishing emails. Int J Hum Comput Stud. 2019;128:17-26. https://doi.org/10.1016/j.ijhcs.2019.02.007

33. Cheek NN, Goebel J. What does it mean to maximize? "Decision difficulty," indecisiveness, and the jingle-jangle fallacies in the measurement of maximizing. Judgm Decis Mak. 2020;15:7-24. 


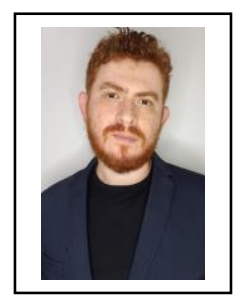

André Luiz de Carvalho Braule Pinto

$\underline{\text { ORCID } \quad \text { Lattes }}$

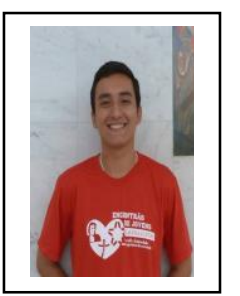

Gabriel dos Santos Mouta

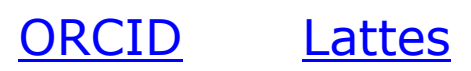

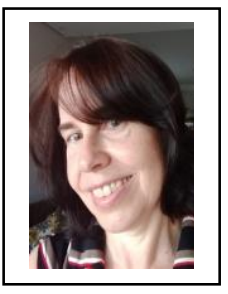

Sonia Regina Pasian

$\underline{\text { ORCID Lattes }}$

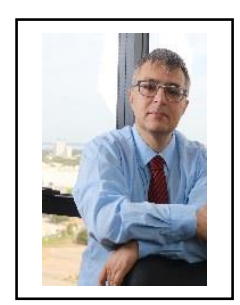

Leonardo Rodrigo Baldaçara

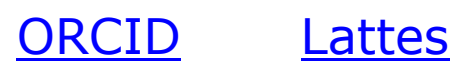

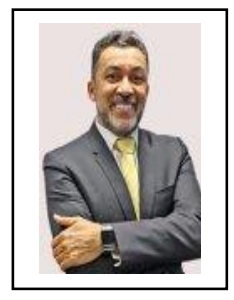

Antônio Geraldo da Silva

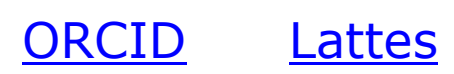

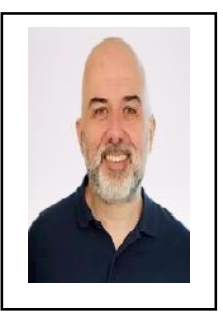

Leandro Fernandes Malloy-Diniz

$\underline{\text { ORCID Lattes }}$ 